\title{
Dynamic equations of motion for a 3-bar tensegrity based mobile robot
}

\author{
Josep M. Mirats Tur, Sergi Hernàndez Juan, Albert Graells Rovira \\ Institut de Robòtica i Informàtica Industrial (IRI) \\ C/Llorens i Artigas 4-6, 08028, Barcelona, Spain \\ jmirats@iri.upc.edu
}

\begin{abstract}
Tensegrity structures can give a new approach to the construction of mobile robots with different shapes and properties that usual robots, wheeled or legged, do not have. Tensegrity are light, deformable structures that may be able to adapt their form to unconstrained environments. The main issue of this paper is to present the dynamic equations of motion for such structures, analysed from a Lagrangian point of view, and thinking in using them as mobile robots of arbitrary form and size.
\end{abstract}

\section{INTRODUCTION}

The word tensegrity is an abreviation for tensile integrity which was coined by Buckminister Fuller in the early 60's [3]. Tensegrity were created by people coming from the art community, [15], being rapidly applied to other disciplines such as in the architectural context, for structures such as geodesic domes [9], or later in space engineering to develop deployable antennas [?]. A general definition for a tensegrity is that of a structure mantaining a stable volume in the space by means of using a discontinuous set of compressive elements which can not vary their length (bars), connected to and by a continuous net of tensile elements which can not increase their lenght (cables or tendons, depending on the application scale) [13]. In fact, there may exist a third kind of element, namely a strut, which can not decrease its lenght, only increase it, but it shall not be considered in the present study. In Fig. 1 some examples of tensegrity structures are given.

So, from an engineering point of view, tensegrity are a special class of structures whose elements may simultaneously perform the purposes of structural force, actuation, sense and feedback control. They have a very high resistance/weight coefficient and are easily deformable. In such kind of structures, theoretically, pulleys or other kind of actuators may stretch/shorten some of the constituting elements in order to substantially change their form with a little variation of the structure's energy. It has been demonstrated that tensegrity structures are very similar to cytoskeleton structures of unicellular organisms [5], [6], some of which are known to move. Tensegrity structures are also very similar to muscle-skeleton structures of high efficiency land animals that can reach speeds up to $60 \mathrm{mph}$. Such animals incorporate tensional elements in their muscle-skeleton system [19] such that they mantain the structure integrity, acting it, and storing and distributing energy [8].
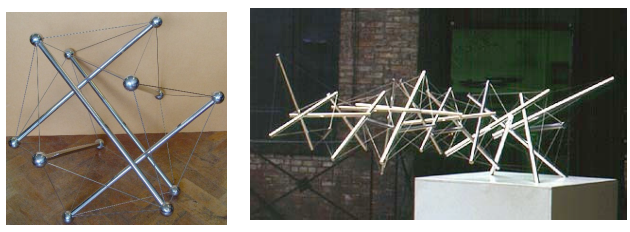

\section{Figure 1. Examples of tensegrity frame- works.}

Due to these similarities with such organisms, we think that tensegrity structures may be a good candidate to construct mobile robots with arbitrary forms and capable of self-deformation in order to adapt efficiently to the environment where they work. Very little attention has been put in tensegrity structures applied to robotics in the literature. Tensegrity structures are used in [1] to obtain redundant manipulator robots that are similar to parallel robots. More recently, in [12], a first try to move such kind of structures in the 3-dimensional space is made by means of a genetic algorithm based control law.

We deal in this paper with the study of the dynamic equations of motion for tensegrity structures thinking in using them to construct mobile robots. The study of the static and dynamic characteristics of such structures has previously received some attention by the scientific community in other areas. Some analytic solutions to the static problem can be found for instance in [2], [11], or [4]. The dynamics of tensegrity were first studied by Motro [10]. In [7], dynamic particle models were studied while considering the bars to be massless; other studies, [14], [16] consider mass on bars. Also non-linear models and their linearization have been considered in [11], [18]. All those studies consider statics and dynamics from an structural point of view (for example, the behaviour of a tensegrity dome under heavy winds), but have not considered the possibility of a tensegriy structure with self-motion 
capability. None of the cited studies has considered, as we present in this work, the six degrees of freedom in the space.

This paper is organised as follows. In Section 2 the general equations of motion for any tensegrity structure are given using Euler-Lagrange formulation. Then, in section 3, a particularization for such equations for the case of a 3-bar tensegrity prism structure are given. Simulations are performed by means of implementing the equations derived in this work. Finally conclusions and future directions are addressed.

\section{General equations of motion}

Consider a generic tensegrity structure with $b$ bars, and hence, $2 b$ nodes, being some of the bars connected by a set of $c$ cables. The purpose of this section is to obtain the general Euler-Lagrange non-linear equations of motion for such structure. Our main interest is to, given the set of external and internal forces, and given the system parameters (i.e., lenghts and physical properties of the tensegrity elements), find the position (trajectory) of the nodes in the 3D-space.

A fixed reference frame, $W$, formed by an orthonormal dextral set of vectors, $(x, y, z)$, is considered such that $z$ points upward and $y$ to the right. The vector of homogeneous coordinates for the $i-t h$ bar will be given by the position of its center of masses, $p_{c m i}=\left(x_{c m i}, y_{c m i}, z_{c m i}\right)^{T}$, with respect to $W$, and three rotations, $s_{i}=\left(\theta_{i}, \psi_{i}, \phi_{i}\right)^{T}$, where $\theta_{i}$ is the inclination angle wih respect to the $x y$ plane, $\psi_{i}$ is the angle of the bar projection onto plane $x y$ with respect to $y$, and $\phi_{i}$ is the angle of rotation of the bar with respect its longitudinal axis. Please, note that physically only two rotations are allowed, $\theta_{i}$ and $\psi_{i}$, while $\phi_{i}$ has to be considered mathematically to prevent the bar orientation to change when changing position. Thus, the vector of homogeneous coordinates for the $i-t h$ bar is denoted as $q_{i}=\left(p_{c m i}, s_{i}\right)^{T}$. For the sake of clarity figure 2 is given.

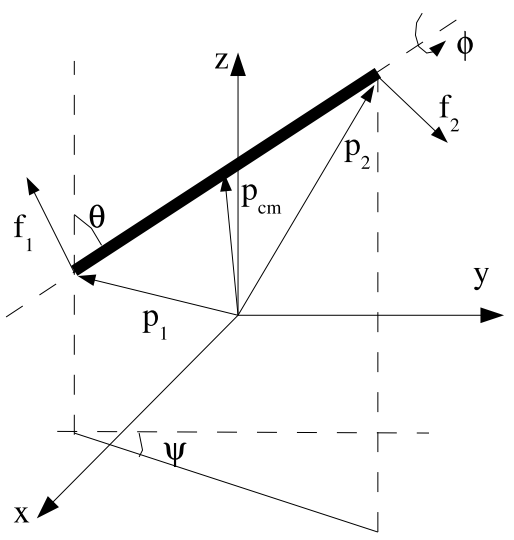

Figure 2. Considered coordinate frame and nodal forces.
Note that the position vector of the $i-t h$ bar corresponding nodes (labelled 1,2 for down and up nodes respectively) with respect to $W$ can be obtained as $p_{1,2 i}=$ $p_{c m i} \mp \frac{l_{i}}{2} \beta_{i}$, where,

$$
\beta_{i}=\left(\begin{array}{c}
\cos \psi_{i} \sin \theta_{i} \\
\sin \psi_{i} \sin \theta_{i} \\
\cos \theta_{i}
\end{array}\right)
$$

Now, the total energy for $i-t h$ bar should be considered. Let $E_{i}$ and $U_{i}$ respectively denote the kinetic and potential energy for the $i-t h$ bar.

$$
E_{i}=\frac{1}{2}\left(m_{i} \dot{p}_{c m i}^{T} \dot{p}_{c m i}+\dot{s}_{i}^{T} I_{i}\left(q_{i}\right) \dot{s}_{i}\right)=\frac{1}{2} \dot{q}_{i}^{T} M_{i}\left(q_{i}\right) \dot{q}_{i}
$$

$$
U_{i}=m_{i} g z_{c m i}=f_{g i}^{T} q_{i}
$$

where $m_{i}$ is the mass of the $i-t h$ bar, $f_{g i}^{T}=$

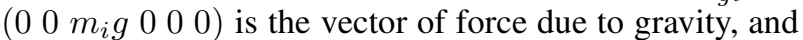
$M_{i}\left(q_{i}\right)$ and $I_{i}\left(q_{i}\right)$ are the generalized mass and inertia moment matrices:

$$
\begin{gathered}
M_{i}\left(q_{i}\right)=\left(\begin{array}{cc}
m_{i} I^{3 x 3} & 0^{3 x 3} \\
0^{3 x 3} & I_{i}\left(q_{i}\right)
\end{array}\right) \\
I_{i}\left(q_{i}\right)=\left(\begin{array}{ccc}
m_{i}\left(\frac{\bar{r}_{i}}{4}+\frac{l_{i}^{2}}{12}\right) & 0 & 0 \\
0 & m_{i}\left(\frac{\bar{r}_{i}}{4}+\frac{l_{i}^{2}}{12}\right) & 0 \\
0 & 0 & \frac{m_{i} r_{i}}{2}
\end{array}\right)
\end{gathered}
$$

Without loss of generality, cylindrical tubular bars have been considered here, being $\bar{r}_{i}=r_{1 i}^{2}+r_{2 i}^{2}$, with $r_{1 i}, r_{2 i}$, the internal and external radius, and $l_{i}$ the lenght of the $i$ - th bar. $I^{n x n}$ represents a $n x n$ identity matrix, and $0^{n x n}$ represents a $n x n$ matrix of zeros.

External forces are considered to be applied on the tensegrity nodes. Using the principle of virtual work the influence of such forces on the total energy of the structure is analysed. Let $j=1,2$ for down and up nodes, then, the virtual work, $\tau_{i}$, exerted on the $i-t h$ bar is given by:

$$
\tau_{i}=\sum_{j} f_{j i} \sum_{k} \frac{\delta p_{j i}}{\delta q_{k i}} \delta q_{k i}
$$

after some operations, the virtual work on the $i-t h$ bar can be expressed as

$$
\tau_{i}=\delta q_{i}^{T} H_{i}\left(q_{i}\right) f_{i}
$$

where $\delta q_{i}^{T}$ is a vector $\left(\delta x_{c m i} \delta y_{c m i} \delta z_{c m i} \delta \theta_{i} \delta \psi_{i} \delta \phi_{i}\right)$ representing the virtual displacement with respect to the generalized coordinates of the bar, $f_{i}=\left(f_{1 i} f_{2 i}\right)$ are 3dimensional $\left(f_{x j i} f_{y j i} f_{z j i}\right)$ vectors of node forces, and $H_{i}\left(q_{i}\right)$ depends on the Jacobian matrix of the force application point position vector with respect to the generalized coordinates of the bar:

$$
H_{i}\left(q_{i}\right)=\left(\begin{array}{cc}
I^{3 x 3} & I^{3 x 3} \\
-J_{i}^{T} & J_{i}^{T}
\end{array}\right)
$$




$$
J_{i}=\left(\begin{array}{ccc}
\frac{l_{i}}{2} \cos \theta_{i} \cos \psi_{i} & \frac{-l_{i}}{2} \sin \theta_{i} \sin \psi_{i} & 0 \\
\frac{l_{i}}{2} \cos \theta_{i} \sin \psi_{i} & \frac{l_{i}}{2} \sin \theta_{i} \cos \psi_{i} & 0 \\
\frac{-l_{i}}{2} \sin \theta_{i} & 0 & 0
\end{array}\right)
$$

Now, considering all the $b$ bars of the tensegrity framework, expressions for the total kinetic and potential energies as well as the total virtual work exerted on the structure by the external forces are given:

$$
\begin{gathered}
E=\sum_{i=1}^{b} E_{i}=\frac{1}{2} \dot{q}^{T} M(q) \dot{q} \\
U=\sum_{i=1}^{b} U_{i}=f_{g}^{T} q \\
\tau=\sum_{i=1}^{b} \tau_{i}=\delta q^{T} H\left(q_{i}\right) f
\end{gathered}
$$

where $q=\left(q_{1} q_{2} \ldots q_{b}\right)^{T}$, is the vector of generalized coordinates of the whole structure, $f_{g}^{T}=\left(f_{g 1} f_{g 2} \ldots f_{g b}\right)$ is the vector of gravity forces, $f=\left(f_{11} f_{21} \ldots f_{2 b}\right)^{T}$ is the $(2 b \times 3) \times 1$ vector of external forces on the nodes, $M(q)$ is a $(b \times 6) \times(b \times 6)$ matrix with block diagonal matrices equal to $M_{i}\left(q_{i}\right)$, and the rest of elements equal to zero, and $H(q)$ is a $(6 \times b) \times(2 b \times 3)$ matrix with block element matrices being $H_{i k}\left(q_{k}\right)$, both $i, k$ spanning from 1 to $b$. These $H_{i k}\left(q_{k}\right)$ matrices take into account the dependency between bars when external forces are applied. Remember that, in a tensegrity, some of the bars are connected to others by means of cables and the resulting forces in each node will in general affect to all the connected bars.

We are now ready to give the general equations of motion for a generic $b$ bar tensegrity structure using the EulerLagrange formulation:

$$
M(q) \ddot{q}+\frac{1}{2}\left(\frac{\delta M^{T}(q)}{\delta q} \dot{q}\right) \dot{q}+f_{g}^{T}=H(q) f
$$

Before concluding this section, two important details concerning the inertia moment matrix given in (4) have to be considered. First, this matrix is usually computed with respect to a coordinates system that is fixed to the bar center of masses, so a linear application has to be used in order to take into account translations and rotations of the bar with respect to the reference frame we are expressing the structure movement. There exists a relation between the fixed and the mobile reference frames of the form $U_{F B}=[S] U_{M B}$, where the subscripts $F B$ and $M B$ denote fixed and mobile base respectively, and $S$ is the matrix that has as columns the components in $F B$ of the $M B$ vectors. After some mathematical manipulation it can be demonstrated that the basis change we are looking for, denoted $W$, relating the mobile base to the fixed one has the form:

$$
W=\left(\begin{array}{ccc}
\cos \phi & 0 & \sin \phi \cos \theta \\
\sin \phi & 0 & -\cos \phi \sin \theta \\
0 & 1 & \cos \theta
\end{array}\right)
$$

Hence, the inertia matrix in the fixed reference frame can be expressed in terms of the inertia matrix with respect to the mobile reference frame, actually the matrix given in (4), using $I_{F B}=W^{T} I_{M B} W$.

Second, note that when the bar is in vertical position this matrix (4) is not invertible so the system will have a singular point. This is a common problem when using spherical coordinates [21] and has two possible solutions: one, to change the axes order when the movement is near to this singular point, and so, accordingly recompute the equations; the other, to work with quaternions [20] and try to see the dynamic problem of motion from a $4 t h$ dimensional space in which this singular point will not exist any more.

\section{3-bar tensegrity equations}

In this section the previous presented general equations are particularized for the case of a 3-bar tensegrity prism. In order to do that, we have to pay special attention to how the external forces are exerted on each node and propose an ordering for the different elements of the structure. Refer to Fig. 3 to a clear insight about the chosen labelling.

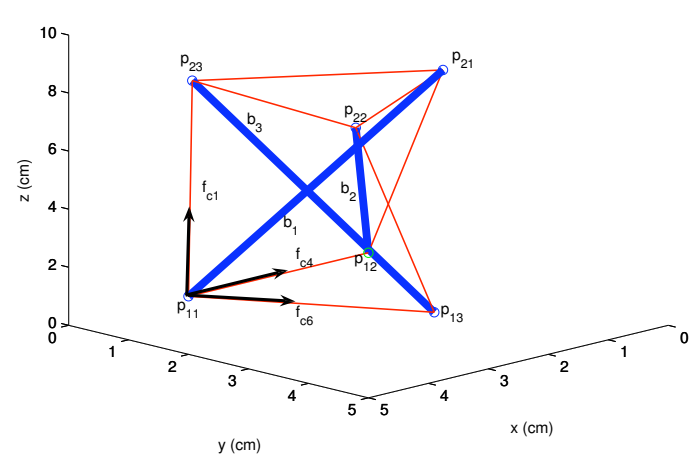

Figure 3. 3-bar tensegrity prism framework.

Equation (6) gives the total virtual work exerted on the $i-t h$ bar by the nodal forces acting on it. For a given structure, each of the $f_{j i}$ terms (remember, force acting on node $j$ of the $i-t h$ bar) can be further decomposed into a sum of forces exerted on the cables connecting each of the bar nodes. For instance, the expression for the force exerted on the lower node of the first bar of the considered structure can be decomposed into $f_{11}=f_{c 1}+f_{c 6}+f_{c 4}$, where the individual terms are given by: 


$$
\begin{aligned}
& f_{c 1}=K_{1}\left(\left\|p_{23}-p_{11}\right\|-L_{1}\right) \frac{p_{23}-p_{11}}{\left\|p_{23}-p_{11}\right\|} \\
& f_{c 6}=K_{6}\left(\left\|p_{13}-p_{11}\right\|-L_{6}\right) \frac{p_{13}-p_{11}}{\left\|p_{13}-p_{11}\right\|} \\
& f_{c 4}=K_{4}\left(\left\|p_{12}-p_{11}\right\|-L_{4}\right) \frac{p_{12}-p_{11}}{\left\|p_{12}-p_{11}\right\|}
\end{aligned}
$$

Please note that previous equations are valid while cables behave as cables, that is, for $\left\|p_{1}-p_{2}\right\|<0$ the force is zero, where $p_{1}$ and $p_{2}$ denote any initial and end point for a considered cable. In these equations, $K_{O}$ represents the stiffness constant for cable $o, L_{o}$ the rest lenght for cable $o$, and $p_{j i}$ the position vector of node $j$ in the $i-t h$ bar with respect to the world coordinates frame $W$. Please, note that the difference between the actual lenght and the rest lenght must always be equal or greater than zero. Now, the previous expression can be easily put into matrix form using $f_{11}=D_{11}\left(q_{1}\right) T_{11}$, where the matrix $D_{11}\left(q_{1}\right)$ contains distances between nodes (cable longitude variations) and $T_{11}$ is the vector of tensions in the cables. Operating the previous expression it is easy to show that:

$$
\begin{gathered}
D_{11}\left(q_{1}\right)=\left[\begin{array}{lll}
\left(p_{23}-p_{11}\right)_{x} & \left(p_{13}-p_{11}\right)_{x} & \left(p_{12}-p_{11}\right)_{x} \\
\left(p_{23}-p_{11}\right)_{y} & \left(p_{13}-p_{11}\right)_{y} & \left(p_{12}-p_{11}\right)_{y} \\
\left(p_{23}-p_{11}\right)_{z} & \left(p_{13}-p_{11}\right)_{z} & \left(p_{12}-p_{11}\right)_{z}
\end{array}\right] \\
T_{11}=\left(\begin{array}{l}
K_{1}\left(1-\frac{L_{1}}{\left\|p_{23}-p_{11}\right\|}\right) \\
K_{6}\left(1-\frac{L_{6}}{\left\|p_{13}-p_{11}\right\|}\right) \\
K_{4}\left(1-\frac{L_{4}}{\left\|p_{12}-p_{11}\right\|}\right)
\end{array}\right)
\end{gathered}
$$

In general, for a tensegrity structure with $b$ bars, there will be $b$ distance matrices, $D_{i}\left(q_{i}\right)$, and $b$ tension vectors, $T_{i}$, that are denoted such as:

$$
D_{i}\left(q_{i}\right)=\left(\begin{array}{cc}
D_{i 1}\left(q_{i}\right) & 0 \\
0 & D_{i 2}\left(q_{i}\right)
\end{array}\right) T_{i}=\left(\begin{array}{c}
T_{1 i} \\
T_{2 i}
\end{array}\right)
$$

For the particular case under study, the 3-bar prismatic tensegrity structure, all the $D_{i}\left(q_{i}\right)$ distance matrices and $T_{i}$ tension vectors are obtained and joined together into a unique $D$ matrix and $T$ vector for the whole structure. In this joint matrix and vector all the distance differences and cable tensions will be duplicated except for a change of sign, depending on the adopted convention on which the tension is defined to be positive. As a general convention, tension is considered to be positive for cables. After some matrix manipulation, it is possible to re-arrange the terms following the designed order for the cables, so the following distance matrix and tension vector are obtained:

$$
D(q)=\left(\begin{array}{ccccccccc}
\mathfrak{a}_{x} & 0 & 0 & \mathfrak{d}_{x} & 0 & \mathfrak{f}_{x} & 0 & 0 & 0 \\
\mathfrak{a}_{y} & 0 & 0 & \mathfrak{d}_{y} & 0 & \mathfrak{f}_{y} & 0 & 0 & 0 \\
\mathfrak{a}_{z} & 0 & 0 & \mathfrak{d}_{z} & 0 & \mathfrak{f}_{z} & 0 & 0 & 0 \\
0 & -\mathfrak{b}_{x} & 0 & 0 & 0 & 0 & -\mathfrak{g}_{x} & -\mathfrak{h}_{x} & 0 \\
0 & -\mathfrak{b}_{y} & 0 & 0 & 0 & 0 & -\mathfrak{g}_{y} & -\mathfrak{h}_{y} & 0 \\
0 & -\mathfrak{b}_{z} & 0 & 0 & 0 & 0 & -\mathfrak{g}_{z} & -\mathfrak{h}_{z} & 0 \\
0 & \mathfrak{b}_{x} & 0 & -\mathfrak{d}_{x} & \mathfrak{e}_{x} & 0 & 0 & 0 & 0 \\
0 & \mathfrak{b}_{y} & 0 & -\mathfrak{d}_{y} & \mathfrak{e}_{y} & 0 & 0 & 0 & 0 \\
0 & \mathfrak{b}_{z} & 0 & -\mathfrak{d}_{z} & \mathfrak{e}_{z} & 0 & 0 & 0 & 0 \\
0 & 0 & -\mathfrak{c}_{x} & 0 & 0 & 0 & 0 & \mathfrak{h}_{x} & -\mathfrak{i}_{x} \\
0 & 0 & -\mathfrak{c}_{y} & 0 & 0 & 0 & 0 & \mathfrak{h}_{y} & -\mathfrak{i}_{y} \\
0 & 0 & -\mathfrak{c}_{z} & 0 & 0 & 0 & 0 & \mathfrak{h}_{z} & -\mathfrak{i}_{z} \\
0 & 0 & \mathfrak{c}_{x} & 0 & -\mathfrak{e}_{x} & -\mathfrak{f}_{x} & 0 & 0 & 0 \\
0 & 0 & \mathfrak{c}_{y} & 0 & -\mathfrak{e}_{y} & -\mathfrak{f}_{y} & 0 & 0 & 0 \\
0 & 0 & \mathfrak{c}_{z} & 0 & -\mathfrak{e}_{z} & -\mathfrak{f}_{z} & 0 & 0 & 0 \\
-\mathfrak{a}_{x} & 0 & 0 & 0 & 0 & 0 & \mathfrak{g}_{x} & 0 & \mathfrak{i}_{x} \\
-\mathfrak{a}_{y} & 0 & 0 & 0 & 0 & 0 & \mathfrak{g}_{y} & 0 & \mathfrak{i}_{y} \\
-\mathfrak{a}_{z} & 0 & 0 & 0 & 0 & 0 & \mathfrak{g}_{z} & 0 & \mathfrak{i}_{z}
\end{array}\right)
$$

$$
T=\left(\begin{array}{lllllllll}
T_{1} & T_{2} & T_{3} & T_{4} & T_{5} & T_{6} & T_{7} & T_{8} & T_{9}
\end{array}\right)^{T}
$$

where, $\mathfrak{a}=\left(p_{23}-p_{11}\right), \mathfrak{b}=\left(p_{21}-p_{12}\right)$, $\mathfrak{c}=\left(p_{22}-p_{13}\right), \mathfrak{d}=\left(p_{12}-p_{11}\right), \mathfrak{e}=\left(p_{13}-p_{12}\right)$, $\mathfrak{f}=\left(p_{13}-p_{11}\right), \mathfrak{g}=\left(p_{21}-p_{23}\right), \mathfrak{h}=\left(p_{21}-p_{22}\right)$, and $\mathfrak{i}=\left(p_{22}-p_{23}\right)$ in order to simplify matrix notation. Matrix $D$, which is a kind of adjacency matrix, carries topological information about the tensegrity structure, not only the actual distances between nodes, but also which nodes are connected to which others, and the pointing direction of each of the cables tension. Note that this matrix has been ordered in the following way: each column represents cables 1 to 9 respectively, and each row stands for bar 1 , node 1 (rows 1 to 3 ), bar 1 node 2 (rows 4 to 6 ), bar 2 , node 1 (rows 7 to 9 ) and so on until bar 3 .

Finally, the expression for the total virtual work exerted by the external forces acting on the bar nodes can be written as:

$$
\tau=\delta q^{T} H(q) D(q) T
$$

where,

$\delta q^{T}=\left(\begin{array}{c}\delta q_{1}^{T} \\ \delta q_{2}^{T} \\ \delta q_{3}^{T}\end{array}\right) H(q)=\left(\begin{array}{ccc}H_{11}\left(q_{1}\right) & 0 & 0 \\ 0 & H_{22}\left(q_{2}\right) & 0 \\ 0 & 0 & H_{33}\left(q_{3}\right)\end{array}\right)$

and the $H_{i i}\left(q_{i}\right)$ are given by (8)

Here, the matrix $H(q)$ carries on information about the transformation between forces and torques in the bars, the matrix $D(q)$ has information about distances between bar nodes that are linked with cables, and vector $T$ gives the information about the tensions in the cables. The product $H(q) D(q)$ is equivalent to the so called equilibrium matrix in [17]. Note that we have obtained an expression which separates the parameters depending on the bars, that is, lenght and center fo mass position, the distances between nodes and the cable tensions. This represents a clear advantage when motion control is to be performed onto the structure. For instance, moving by cable control 
is easily achieved into the equations by only adding a term to each of the $T$ components vector of the form:

$$
T_{c o}=K_{o} \frac{u_{c o}}{\|\cdot\|}
$$

where $u_{c o}$ is the control order which must fulfill the conditions $L_{o}+u_{c o} \leqslant L_{\text {break }}$ and $L_{o} \geqslant u_{c o}$. Note that $u_{c o}$ may be negative indicating a cable stretching.

\section{Simulation}

We present in this section the results of simulating the movement of a 3-bar tensegrity structure in the 3D-space by means of using the developed dynamical equations. The simulation is provided in order to check the validity of the presented equations when compared to the real movement of the structure. An initial position is considered such that one of the vertices of the triangular bottom side is given a positive $z$, so the structure will fall down because of the gravity acting on its elements. Figure 4 shows a caption from the simulator, where the tensegrity is in the described initial position.

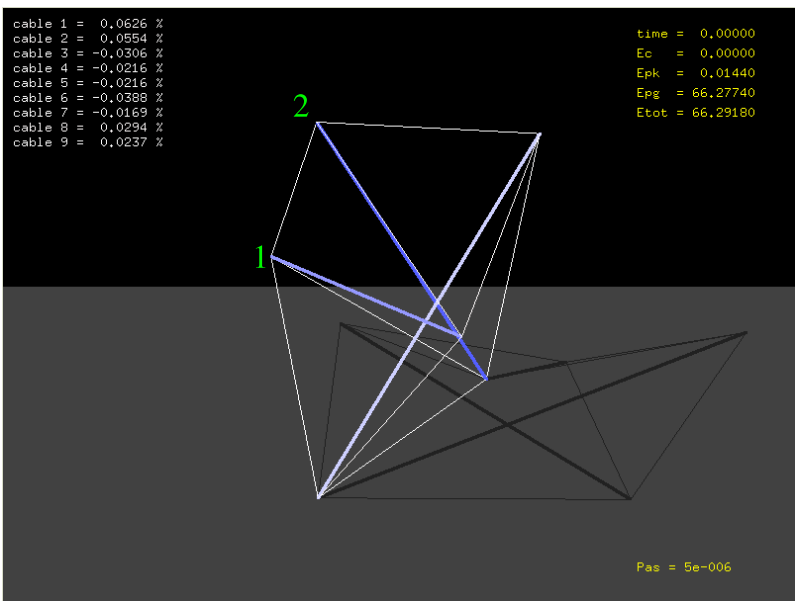

Figure 4. Initial position for the movement simulation of a 3-bar tensegrity prism.

Figures 5 and 6 show the change on $(x, y, z)$ coordinates for labelled nodes 1 and 2 in figure 4. Employed units in both figures are centimeters. Also, together with the written paper we attached a video where the simulation of the described movement can be appreciated. It can be downloaded from http://haydn.upc.es/people/jmirats/publicacions.html

\section{Conclusions and future work}

We have considered in this work the possibility of creating mobile robots by means of using tensegrity structures. As a first step we have designed the general equations of motion for such structures in the three dimensional space using the Euler-Lagrange formulation. Then,
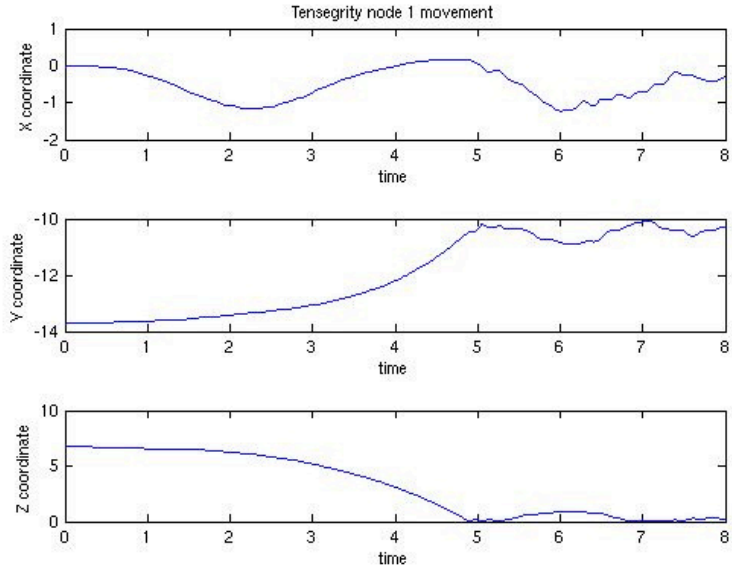

Figure 5. Node 1 movement. Variation of its $(x, y, z)$ coordinates
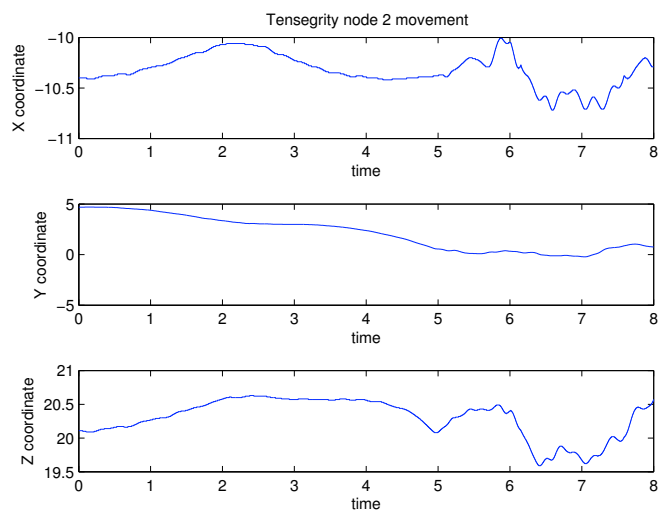

Figure 6. Node 2 movement. Variation of its $(x, y, z)$ coordinates

we particularized these equations of motion for a 3-bar tensegrity structure. Interesting results are found, when in (14) an expression is obtained which separates the parameters depending on the bars, the distances between nodes and the cable tensions. This represents a clear advantage when motion control is to be performed onto the structure, as is the case of mobile robotics. Authors are now developing a prototype of mobile robot that will be able to move thanks to controlling the changes in the cable lenghts. During the presented study, energy conservation has been adopted as hypothesis, which is not a realistic assumption, hence the dynamic equations of motion developed in this work have to be updated taking into consideration energy loss due to kinetic friction when the structure is moving. 


\section{Acknowledgements}

This work has been partially supported by projects DPI2006-14001 and Profit CIT-020400-2007-78 both financed by the Spanish Education and Science Ministry.

\section{References}

[1] J. Aldrich. Control synthesis for a class of light and agile robotic tensegrity structures. $\mathrm{PhD}$ thesis, University of California, 2004.

[2] J. Correa. Static analysis of tensegrity structures. Master's thesis, Univ. of Florida, 2001.

[3] R. Fuller. Tensile-integrity structures. United States Patent 3063521, November 1962.

[4] M. K.-A. H. Kebiche and R. Motro. Geometrical nonlinear analysis of tensegrity systems. Eng. Struct., 21:864876, 1998.

[5] D. Ingber. Cellular tensegrity: defining new rules for biological design that govern the cytoskeleton. Journal of Cell Science, 104:613-627, 1993.

[6] D. Ingber. Architecture of life. Scientific American, 52:4857, 1998.

[7] N. Kanchanasaratool and D. Williamson. Modelling and control of class nsp tensegrity structures. Int. J. Control, 75:123-139, 2002.

[8] S. Levin. The tensegrity-truss as a model for spinal mechanics: Biotensegrity.

[9] R. Motro. Tensegrity systems and geodesic domes. Int. Journal of space structures, 5(3-4):341-351, 1992.

[10] R. Motro, S. Najari, and P. Jouanna. Static and dynamic analysis of tensegrity systems. In Proceedings of the ASCE International Symposium on Shell and Spatial Structures: Computational aspects, pages 270-279. Springer, 1986.

[11] H. Murakami and Y. Nishimura. Static and dynamic characterization of regular truncated icosahedral and dodecahedral tensegrity modules. International Journal of Solids and Structures, 38(50-51):9359-9381, 2001.

[12] C. Paul, J. Roberts, H. Lipson, and F. Cuevas. Gait production in a tensegrity based robot. In Proceedings of the 2005 International Conference on Advanced Robotics (ICAR), Seattle, Washington, USA, July 18th-20th., 2005.

[13] A. Pugh. An introduction to tensegrity. University of California Press, 1976.

[14] R. Skelton, J. Pinaud, and D. Mingori. Dynamics of the shell class of tensegrity structures.

[15] K. Snelson. Continuous tension, discontinuous compression structures. United States Patent 3169611, February 1965.

[16] C. Sultan, M. Corless, and R. Skelton. Reduced prestressability conditions for tensegrity systems. Proc. of 40th AIAA/ASME/ASCE/AHS/ASC Structures, Structural Dynamics and Materials Conf., 1999, St. Louis (MO), 1999.

[17] C. Sultan, M. Corless, and R. Skelton. The prestressability problem of tensegrity structures: some analytical solutions. Journal of Solids and Structures, 38:5223-5252, 2001.

[18] C. Sultan, M. Corless, and R. Skelton. Linear dynamics of tensegrity structures. Engineering Structures, 24(6):671$685,2002$.

[19] S. Timoshenko and D. Young. Cats' Paws and Catapults: Mechanical Worlds of Nature and People. W. W. Norton and Company, 2000.
[20] J. Ward. Quaternions and Cayley Numbers. Kluwer Academic Publishers, 1997.

[21] Wikipedia. Gimbal lock, the free encyclopedia, http://en.wikipedia.org/w/index.php?title=gimbal_lock\&oldid=57860768. 